\title{
Calibration of CR-39 solid state track detectors with monoenergetic protons from 0.3 MeV to $2.5 \mathrm{MeV}$
}

\author{
Yangfan $\mathrm{He}^{1,2, *}$, Xiaofeng $\mathrm{Xi}^{1, *}$, Shilun $\mathrm{Guo}^{1}$, Bing Guo ${ }^{1, * *}$, Changye $\mathrm{He}^{1}$, Fulong Liu ${ }^{1,3}$, Xiaofei Lan ${ }^{2}$, Menglin Qiu ${ }^{3}$, \\ Guangfu Wang $^{3}, D i \mathrm{Wu}^{1}$, Jihong Wei ${ }^{1,3}$, Luohuan Wang ${ }^{1,3}$, Wansha Yang ${ }^{1,3}$, and Donghai Zhang ${ }^{4}$ \\ ${ }^{1}$ Department of Nuclear Physics, China Institute of Atomic Energy, Beijing 102413, China \\ ${ }^{2}$ School of Physics and Space Sciences, China West Normal University, Nanchong 637002, China \\ ${ }^{3}$ College of Nuclear Science and Technology, Beijing Normal University, Beijing 100875, China \\ ${ }^{4}$ School of Physics and Information Engineering, Shanxi Normal University, Linfen 041004, China
}

\begin{abstract}
The ${ }^{2} \mathrm{H}(\mathrm{d}, \mathrm{p})^{3} \mathrm{H}$ reaction is one of the most crucial reactions in the Big Bang nucleosynthesis (BBN). It is of particular interest to investigate this kind of reactions in plasma environments, generated by high intensity lasers, which are similar to real astrophysical conditions. We have experimentally investigated the ${ }^{2} \mathrm{H}(\mathrm{d}, \mathrm{p})^{3} \mathrm{H}$ reaction using laser-driven counter-streaming collisionless plasmas at the Shenguang-II laser facility. CR-39 track detectors are widely employed as the main diagnostics in such experiments and laser-driven ion acceleration. In this work, we performed calibration of CR-39 track detectors with monoenergetic protons from the tandem accelerator, and then presented their track diameters for proton energies ranging from $300 \mathrm{keV}$ to 2.5 $\mathrm{MeV}$ and for etching times between 4 and 28 hours. In addition, we recommended the optimal etching time at the typical etching conditions, which will be very useful for the following massive data analysis from the CR-39 detectors.
\end{abstract}

\section{Introduction}

Nuclear fusions are the most crucial reactions in nuclear astrophysics because they are responsible not only for powering stars but also for the synthesis of the elements in the universe $[1,2]$. The ${ }^{2} \mathrm{H}(\mathrm{d}, \mathrm{p})^{3} \mathrm{H}$ fusion reaction plays a key role not only in the design of future fusion power plants but also in the understanding of primordial abundances in Big Bang nucleosynthesis (BBN) models [3]. Therefore, this reaction has been studied using accelerators for many decades (see [4] and references therein). It is of particular interest to investigate this kind of reactions in plasma environments, generated by high intensity lasers, which are close to real astrophysical conditions. In recent years, with the rapid development of high-intensity laser technology, it is possible to produce this kind of plasma environment in the laboratories [5-9].

Recently we have performed experimental investigation of the ${ }^{2} \mathrm{H}(\mathrm{d}, \mathrm{p})^{3} \mathrm{H}$ reaction using laser-driven counterstreaming collisionless plasmas at the Shenguang-II laser facility at Shanghai Institute of Optics and Fine Mechanics of Chinese Academy of Sciences. In such a measurement, identification of reaction products and determination of their number have some difficulties, such as the electromagnetic pulse which can obstruct electronic devices. The CR-39 solid state track detectors $[10,11]$ are widely employed as the main diagnostics in the fusion reaction experiments [12] and ion acceleration by superintense lasers

\footnotetext{
* These authors contributed equally to this work.

**Corresponding author: guobing@ ciae.ac.cn
}

[13] because they are mostly sensitive to ions but are insensitive to the backgrounds such as electrons and photons. When charged particles collide with a sheet of CR-39 solid state track detectors, they generate the tracks observable with an optical microscope after an etching stage under controlled conditions. The detection efficiency of CR39 was found to be nearly $100 \%$ for ion energies higher than $100 \mathrm{keV}$ [14]. It was also demonstrated that the energy threshold could be around $20 \mathrm{keV}$ [15-17]. We used CR-39 solid state track detectors to record the energy and number of the protons generated by the ${ }^{2} \mathrm{H}(\mathrm{d}, \mathrm{p})^{3} \mathrm{H}$ reaction. Proton identification requires the information on the track's diameters, depths and patterns which primarily depend on the proton energy, the etching parameters (solution temperature and concentration), and the etching time. However, the performance of CR-39 track detectors varies from batch to batch in the same factory [18]. Thus, one must calibrate the same batch of the CR-39 detectors with mono-energetic protons as that used in study of fusion reactions. To date the calibration of CR-39 solid state detectors with proton beam has several methods such as Rutherford backscattering method [19, 20], nuclear reaction method [21], laser accelerated proton (TNSA acceleration mechanism) combined with mass spectrometer [22], beam irradiation $[17,23]$.

In this work, we aim at calibration of CR-39 detectors with monoenergetic protons from $2 \times 1.7 \mathrm{MV}$ tandem accelerator, using a rotating target plate with CR-39 pieces pasted on it to receive the irradiation below required density. 
Table 1. Proton energy and the used CR-39 sheet number for each round in our calibrations.

\begin{tabular}{cc}
\hline Energy (MeV) & Number of CR-39 \\
\hline 2.5 & 15 \\
2.3 & 13 \\
2.1 & 13 \\
1.9 & 13 \\
1.7 & 13 \\
1.5 & 14 \\
1.3 & 13 \\
1.1 & 13 \\
0.9 & 13 \\
0.7 & 13 \\
0.5 & 13 \\
0.3 & 15 \\
\hline
\end{tabular}

\section{Experimental procedure}

The proton beam was delivered by GIC4117 tandem accelerator at Beijing Normal University. In the experiment, proton beam intensity was about $20 \mathrm{pA}$ during the calibration at different energies. A schematic layout of the experimental setup is shown in Fig. 1.

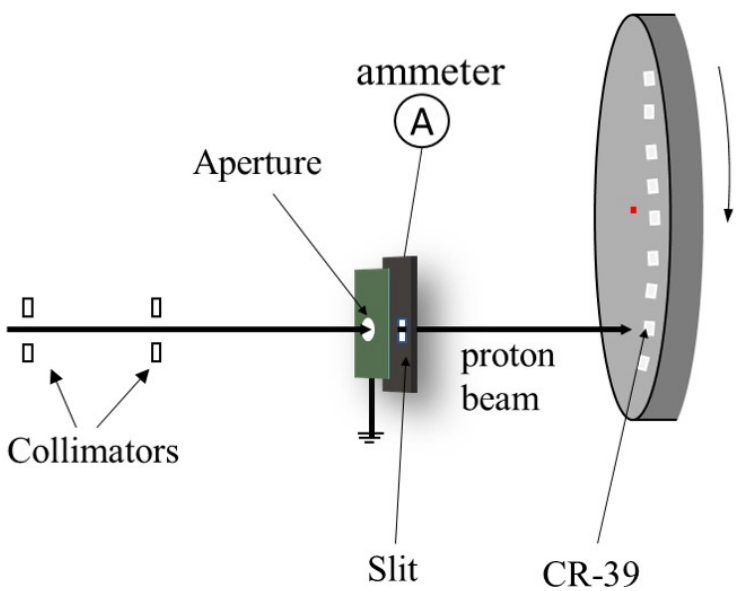

Figure 1. Schematic Layout of experimental setup.

In Fig. 1, two collimators are placed on the path where proton beam comes from. An aperture with the diameter of the $1 \mathrm{~cm}$ and a slit with the width of $0.439 \mathrm{~mm}$ are used to decrease the beam intensity. We connected the slit to an ammeter to get the beam intensity. CR-39 solid state track detector (Fukuvi Chemical Industry, Tokyo, Japan) sheets are pasted on a big rotating plate and arranged in a circle. For each CR-39 sheet, its geometrical dimension is $10 \mathrm{~mm}$ $\times 10 \mathrm{~mm} \times 0.9 \mathrm{~mm}$. The maximum speed of the rotating plate is $109 \mathrm{rpm}$.

In each measurement, the controllable target plate rotated for only one time, so the duration of irradiation on a CR-39 detector is the period of time that proton beam shots across one CR-39 sheet. This irradiating duration was measured by a He-Ne laser. The laser passed through the slit and received by an oscilloscope. The irradition time on each CR-39 piece is measured to be $12.5 \mathrm{~ms}$. It can be estimated that the amount of protons irradiated on one CR-39 solid state detector is $\sim 5 \times 10^{4}$. Table 1 lists out the proton energies, number of the used CR-39 pieces for each round.

The irradiated CR-39 sheets were chemially etched in the $6.5 \mathrm{~mol} / \mathrm{L}$ of $\mathrm{NaOH}$ solution at the temperature of $70 \pm 0.1^{\circ} \mathrm{C}$, and the volume of solution didn't change when we finished etching. The etched CR-39 sheets were processed in six steps: (1) Wash in $700 \mathrm{ml}$ of deionized water for 5 minutes; (2) Wash in $500 \mathrm{ml}$ of deionized water for 5 minutes and then wash for the second time; (3) Wash in $250 \mathrm{ml}$ dilute nitric acid (10\%) for 5 minutes;(4) Soak in $600 \mathrm{ml}$ of deionized water for 5 minutes; (5) Soak in absolute ethanol (analytical purity) for 5 minutes; (6) Put it in a petri dish and then put them in a self-sealing bag.

The proton track diameters were measured by an automatic image analyzer provided by Beijing Institute of Radiology Medicine [25]. The optical images from microscope were acquired as analog pictures with CCD camera, then analog pictures were converted into digital ones by image acquisition card, and thus the track diameters were measured by image processing. In our experiment, the proton beam was focused on the CR-39 sheets at normal incidence. Therefore, the diameter of the track can be used as a reliable parameter for the calibration.

\section{Results}

Some sample tracks which were etched for 4 hours are shown in Fig. 2. In Fig. 3, we plot the proton track diameter versus proton energy etched for different times ( $4 \mathrm{~h}, 8$ $\mathrm{h}, 12 \mathrm{~h}, 16 \mathrm{~h}, 20 \mathrm{~h}, 24 \mathrm{~h}, 28 \mathrm{~h}$ ) at $70^{\circ} \mathrm{C}$ in the $6.5 \mathrm{~mol} / \mathrm{L}$ of $\mathrm{NaOH}$. Two preliminary conclusions can be drawn from Fig. 3: (1) Long time etching will make the track of lowenergy protons shallow and difficult to measure accurately, hence the etching duration of the CR-39 detectors should not be too long; (2) The longer time of etching, the higher energy of the peak will move to for the curves of track diameter vs proton energy. Under the present etching conditions described herein, the tracks diameter of $0.3 \mathrm{MeV}$ protons was the largest for 4 hours of etching. With the etching time increasing to 28 hours, the proton energy for the maximum value of track diameter changes to $1.1 \mathrm{MeV}$.

\section{Summary and conclusion}

In summary, we have carried out the proton calibration for CR-39 solid state track detectors which have been used in our study of the laser-driven ${ }^{2} \mathrm{H}(\mathrm{d}, \mathrm{p})^{3} \mathrm{H}$ reaction. The track diameters were given for the proton energies from $0.3 \mathrm{MeV}$ to $2.5 \mathrm{MeV}$ (12 energy points) and for seven different etching times from 4 hours to 28 hours. Finally, we recommended the optimal etching times to be 8-20 hours at the typical etching conditions of $70^{\circ} \mathrm{C}$ in $6.5 \mathrm{~mol} / \mathrm{L}$ $\mathrm{NaOH}$. The present calibration results will be very helpful for identifying the reaction products emitted from the ${ }^{2} \mathrm{H}(\mathrm{d}, \mathrm{p}){ }^{3} \mathrm{H}$ fusion reaction.

\section{Acknowledgment}



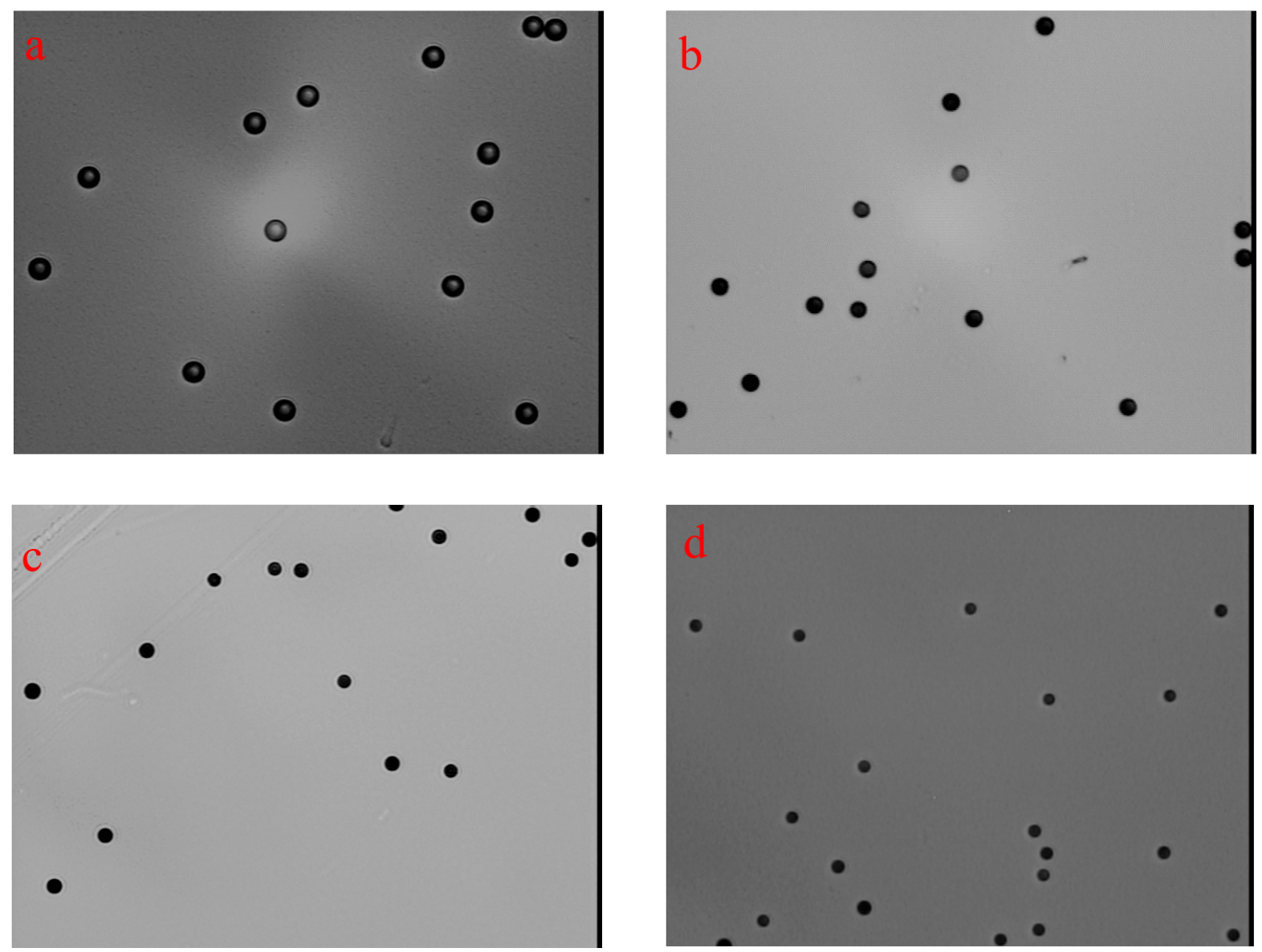

Figure 2. Microphotographs of tracks from normally incident protons: a) $0.3 \mathrm{MeV}$ protons ( 4 h chemical etching); b) $0.5 \mathrm{MeV}$ protons (4 h chemical etching); c) $1.5 \mathrm{MeV}$ protons (4 h chemical etching) and d) $1.9 \mathrm{MeV}$ protons (4 $\mathrm{h}$ chemical etching).

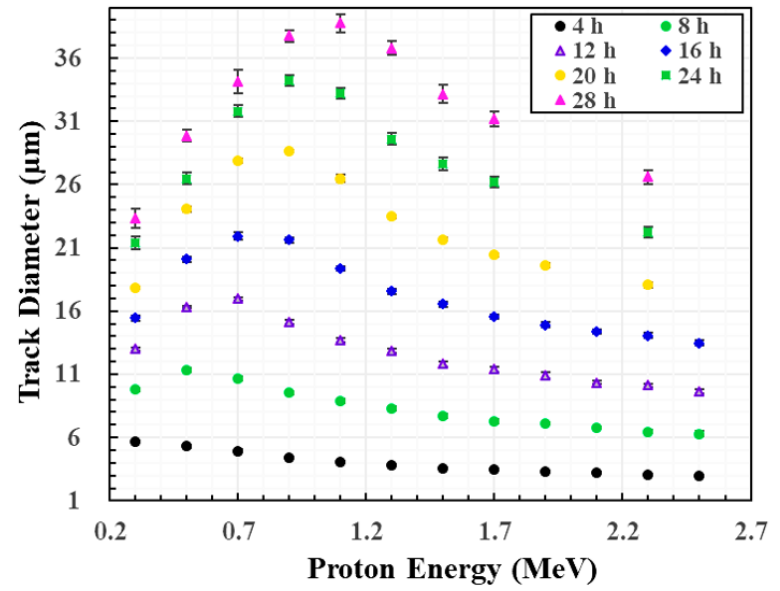

Figure 3. Track diameter vs. proton energy.

The authors would like to thank Shengyong Su, Shaozhe Zhang, Xiaohao Meng and Chen Wang for their helpful comments. This work was supported by the National Key Research and Development Project under Grant No. 2016YFA0400502, the National Natural Science
Foundation of China under Grants No. 11775312 and No. 11975316.

\section{References}

[1] E.M. Burbidge, G.R. Burbidge, W.A. Fowler and F. Hoyle, Rev. Mod. Phys. 29, 547 (1957).

[2] G. Wallerstein, I.I. Jr., P. Parker et al., Rev. Mod. Phys. 69, 995 (1997).

[3] Richard H. Cyburt, Brian D. Fields, Keith A. Olive and Tsung-Han Yeh, Rev. Mod. Phys. 88, 015004 (2016).

[4] Y. Xu, K. Takahashi, S.Goriely et al., Nucl. Phys. A 918, 61 (2013).

[5] L. Robson, P. Simpson, R.J. Clarke et al., Nat. Phys. 3, 58 (2007).

[6] P. McKenna, K. Ledingham, T. McCanny et al., Phys. Rev. Lett. 91, 075006 (2003).

[7] L. Torrisi, Radiation Effects and Defects in Solids 172, 61 (2017).

[8] C. Labaune, C. Baccou, V. Yahia, C. Neuville, J. Rafelski, Sci. Rep. 6, 21202 (2016).

[9] V. Belyaev, A. Matafonov, V. Vinogradov et al., Phys. Rev. E 72, 026406 (2005). 
[10] R. Fleischer, P. Price, R. Walker, J. Appl. Phys. 36, 3645 (1965).

[11] B.G. Cartwright, E. Shirk, P. Price, Nucl. Instrum. Methods 153, 457 (1978).

[12] C. Labaune, C. Baccou, S. Depierreux et al., Nat. Commun. 4, 2506 (2013).

[13] A. Macchi, M. Borghesi, M. Passoni, Rev. Mod. Phys. 85, 751 (2013).

[14] S. Gaillard, J. Fuchs, N. Renard-Le Galloudec, and T. E. Cowan, Rev. Sci. Instrum. 78, 013304 (2007).

[15] E. Skladnik-Sadowska, J. Baranowski, and M. Sadowski, Radiat. Meas. 34, 337 (2001).

[16] K. Malinowski, E. Skladnik-Sadowska, and M. J. Sadowski, Radiat. Meas. 40, 371 (2005).

[17] X.J. Duan, X.F. Lan, Z.X.Tan et al., Nucl. Instrum. Methods Phys. Res. A 609, 190 (2009).
[18] E. Vilela, E. Fantuzzi, G. Giacomelli et al., Radiat. Meas. 31, 437 (1999).

[19] L. Bernardi, A. Cecchi, C. Gori, F. Lucarelli, R. Renzi, Nucl. Instrum. Methods Phys. Res. B 53, 61 (1991)

[20] Z. Lounis, S. Djeffal, K. Morsli, and M. Allab, Nucl. Instrum. Methods Phys. Res. B 179, 543 (2001).

[21] N. Sinenian, M. Rosenberg, M. Manuel et al., Rev. Sci. Instrum. 82, 103303 (2011).

[22] C. Baccou, V. Yahia, S. Depierreux et al., Rev. Sci. Instrum. 86, 083307 (2015).

[23] Y. Zhang, H.W. Wang, Y.G. Ma et al., Nucl. Sci. Tech. 30, 87 (2019).

[24] X.J. Duan, Z.X. Tan, X.F. Lan et al., Acta Physica Sinica 59, 3147 (2010) (in Chinese).

[25] Y.S. Luo, Y. Zhou, X.G. Wang, Nucl. Tech. 25, 541 (2002). 